\title{
Does the conventional landmark help to place the tip of REBOA catheter in the optimal position? A non-controlled comparison study
}

Kento Nakajima ${ }^{1,2^{*}}$ (D), Hayato Taniguchi ${ }^{2,3}$, Takeru Abe ${ }^{1,2}$, Keishi Yamaguchi ${ }^{1,2}$, Tomoki Doi ${ }^{2,4}$, Ichiro Takeuchi ${ }^{1,2}$ and Naoto Morimura ${ }^{5}$

\begin{abstract}
Background: Resuscitative endovascular balloon occlusion of the aorta (REBOA) for patients with traumatic torso hemorrhagic shock is available to keep a minimum level of circulatory status as a bridge to definitive therapy. However, the trajectory for placement of REBOA in the aorta has not yet been clearly defined.

Methods: We conducted a retrospective observational cohort study in the two tertiary critical care and emergency center from December 2014 to October 2018. A total of 28 patients who underwent focused assessment with sonography for trauma (FAST) were studied via contrast computed tomography (CT), and 27 were analyzed.

Results: We divided patients into two groups based on our $C T$ findings. The REBOA deflate group included 16 patients, and the inflate group included 11 patients. The median trace value (interquartile range) of the blood vessel center line from the common femoral artery to the tip of REBOA (blood vessel length) and the length of REBOA itself from the common femoral artery to the tip of REBOA (REBOA insertion length) were $56.2 \mathrm{~cm}(54.5-$ $57.2)$ and $55.2 \mathrm{~cm}(54.2-55.6)$, respectively $(p<0.0001)$ for the deflated group, and $51.4 \mathrm{~cm}(42.1-56.6)$ and $50.3 \mathrm{~cm}$ $(42.3-55.0)(p=0.594)$, respectively, for the inflated group.

Conclusions: If REBOA was deflated, it was placed $1.0 \mathrm{~cm}$ longer than the insertion length of REBOA catheter itself, but that was not the case when inflating REBOA. The individual difference was large to the extent that the balloon inflated and the extent to which the balloon was pushed back toward the caudal depending on the degree of blood pressure. Further studies would be needed to validate the study findings.
\end{abstract}

Keywords: Torso trauma, Hemorrhagic shock, Resuscitative endovascular balloon occlusion of the aorta

\section{Background}

Hemorrhagic shock is a major cause of traumatic death [1-7]. To avoid trauma death, it is important to stop bleeding as soon as possible. Resuscitative endovascular balloon occlusion of the aorta (REBOA) for patients with traumatic torso hemorrhagic shock is available to keep a

\footnotetext{
* Correspondence: nakajimak.812@gmail.com

${ }^{1}$ Advanced Critical Care and Emergency Center, Yokohama City University Medical Center, 4-57 Urafunecho, Minami-ku, Yokohama, Kanagawa 232-0024, Japan

2Department of Emergency Medicine, Graduate School of Medicine, Yokohama City University, 3-9 Fukuura, Kanazawa-ku, Yokohama, Kanagawa 236-0004, Japan

Full list of author information is available at the end of the article
}

minimum level of circulatory status as a bridge to definitive therapy $[2,8-12]$.

Especially for profound shock patients, the prompt placement of REBOA is essentially important. However, in such situations, there are limitations of time and equipment to accurately place REBOA, which is ideally placed under fluoroscopy. To date, using the midsternum as a landmark, and inserting REBOA, the length from the thigh to the mid-sternum is implied in the range of aortic zone I [13]. If the insertion length of REBOA is longer than the length from the thigh to the xiphoid process in the body surface and is shorter than the length from the thigh to the sternum notch, the tip

(c) The Author(s). 2019 Open Access This article is distributed under the terms of the Creative Commons Attribution 4.0 International License (http://creativecommons.org/licenses/by/4.0/), which permits unrestricted use, distribution, and 
is placed in the aortic zone I [14]. In addition, morphometric roadmaps have been identified to keep REBOA in an exact zone under non-fluoroscopy [15].

However, the trajectory of where to place REBOA in the aorta has not been clearly defined [16]. For example, it is expected that the position of the tip is different from the estimated value on the desk due to inflation or deflation of the balloon, circulation dynamics, and the like. There is no margin of time to consider during the resuscitation of patients with severe trauma, and an indicator to predict how close to the target zone REBOA will reach under non-fluoroscopy is required. Thus, in this study, we identified how REBOA traveled through the aorta and where the tip was located, and how much it deviated from the estimated value in the reconstructed computed tomography (CT). Our study hypothesis was that blindly but safely placed REBOA in the targeted zone can be inserted at different distances depending on whether REBOA is inflated or deflated. Our findings can enable practitioners to obtain a more precise REBOA placement distance, consequently leading to a safer approach that is unaffected by institutional or personnel variability.

\section{Methods}

\section{Patients and study setting}

This was a retrospective observational cohort study. We targeted trauma patients who underwent focused assessment with sonography for trauma (FAST) and transported to Yokohama City University Medical Center's Advanced Critical Care and Emergency Center, in Yokohama City (YCU), and Yokosuka Kyosai Hospital Critical Care and Emergency Center, in Yokosuka City (YKH), Japan, from December 2014 to October 2018. The study was approved by the institutional review boards at both institutes.

The population of Yokohama City was 3,740,944 in 2019 [17], and there are nine critical care and emergency centers in the city. The population covered per emergency medical center would be approximately 415,660. Yokosuka City has two emergency medical centers, and those centers would be responsible for critical patients in Yokosuka City, adjacent Miura City, and Zushi City. The population of Yokosuka, Zushi, and Miura City was 497,452 from the latest data [18-20]. Thus, the population covered per emergency medical center would be approximately 248,726 .

In the study institutions, the availability and immediacy of trauma surgeons and interventional radiology (IVR) physicians could vary the time until radical hemostasis. In addition, the length of time until a portable X-ray can be utilized and CT imaging can be conducted for each patient might also differ. In cases of shock due to severe trauma, without critical positive findings in head or chest trauma and with suspected bleeding in the abdominal or retroperitoneal cavity,
REBOA can be placed in aortic zone I. In such cases, deflated REBOA could be used as a bridge to definitive hemostasis. In cases of negative FAST and intraabdominal hemorrhage, REBOA can be placed in aortic zone III. However, to rapidly place REBOA under nonfluoroscopy, it can initially be positioned in the wider aortic zone I. After CT imaging and diagnosis, the placement position can be changed to aortic zone III depending on treatment necessity.

The exclusion criteria were patients under 15 years of age, without FAST enforcement, without REBOA insertion, without CT imaging, or only simple CT imaging. The patients with REBOA which did not reach aortic zone III were excluded. Of the 1897 patients in two facilities, and among the 76 patients in whom REBOA was inserted, a total of 28 patients had taken a contrast CT. The inserted length of REBOA itself and the center line of the blood vessel from the common femoral artery to the tip of REBOA would be equivalent to the blood vessel length. We excluded one patient whose tip of REBOA did not reach aortic zone III in threedimensional (3D) medical images, and analyzed 27 patients (Fig. 1). Measurement was performed using data processing software (Ziostation 2 PLUS, Ziosoft Corp., Tokyo, Japan). We plotted the center point of the contrasted vascular lumen from the common femoral artery to the blood vessel cross section at the tip of REBOA at the horizontal disconnection of CT. Then, we reconstructed meandering blood vessels approximately linearly and measured the blood vessel length (Fig. 2). In this study, systolic blood pressure of $90 \mathrm{mmHg}$ or less was defined as low blood pressure [2, 21].

\section{Indication and procedure}

The aorta is classified into three zones for the purpose of REBOA insertion. Aortic zone I extends from the origin of the left subclavian artery to the celiac artery. Aortic zone II extends from the celiac artery to the lowest renal artery. Aortic zone III exists from the lowest renal artery to the aortic bifurcation (Fig. 3) [12, 22]. We also defined a zone which exceeded aortic zone I as aortic zone 0 . REBOA is mainly placed in aortic zone I for intra-abdominal bleeding control and in aortic zone III for pelvic fracture and control of lower limb bleeding. However, in emergency situations, REBOA is often placed in aortic zone I for the time being [2, 23-25].

In patients requiring REBOA, we first inserted a 4- to 6-Fr short percutaneous sheath from the common femoral artery and exchanged it to a 7-Fr short sheath [26]. The femoral artery is the most common access site [21]. Next, the guide wire was advanced, and REBOA was placed blindly under non-fluoroscopy by the emergency physician. We used a small-diameter 7-Fr Rescue 


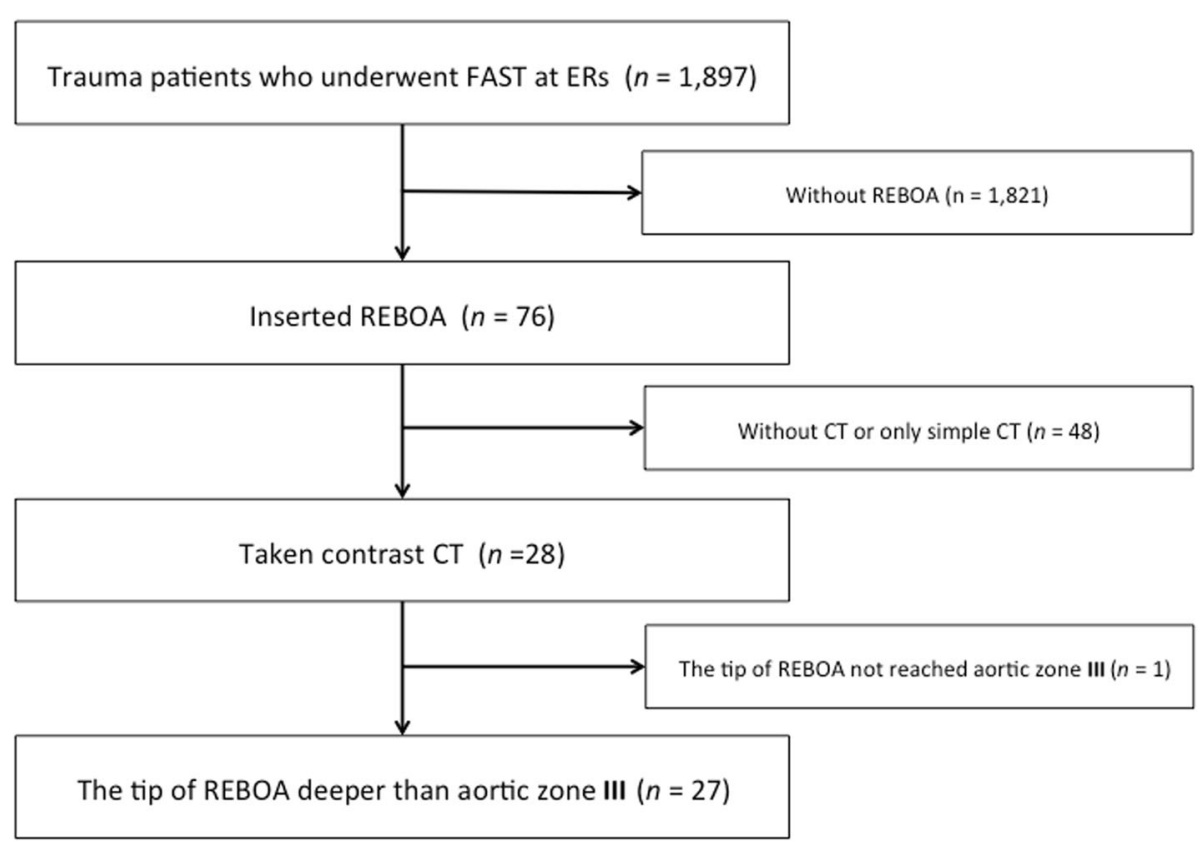

Fig. 1 Flow chart of patient inclusion in this study. Of the 1897 patients in two facilities, and among the 76 patients in whom REBOA was inserted, a total of 28 patients had taken a contrast $C T$. The inserted length of REBOA itself and the center line of the blood vessel from the common femoral artery to the tip of REBOA would be equivalent to the blood vessel length. We excluded one patient whose tip of REBOA did not reach aortic zone III and analyzed 27 patients

a

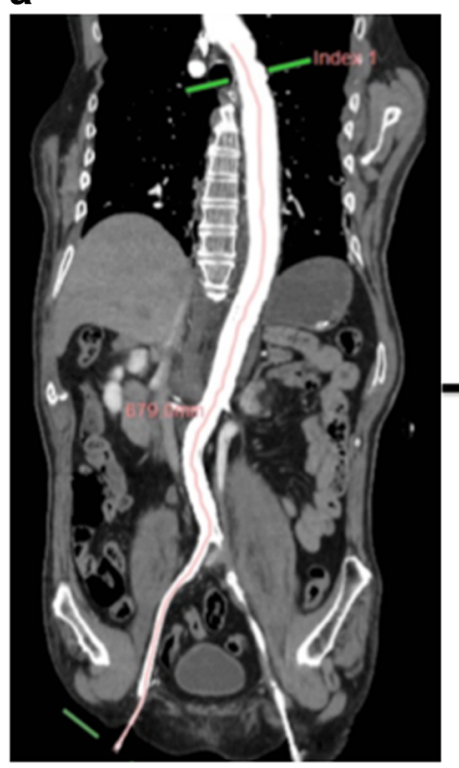

\section{b}

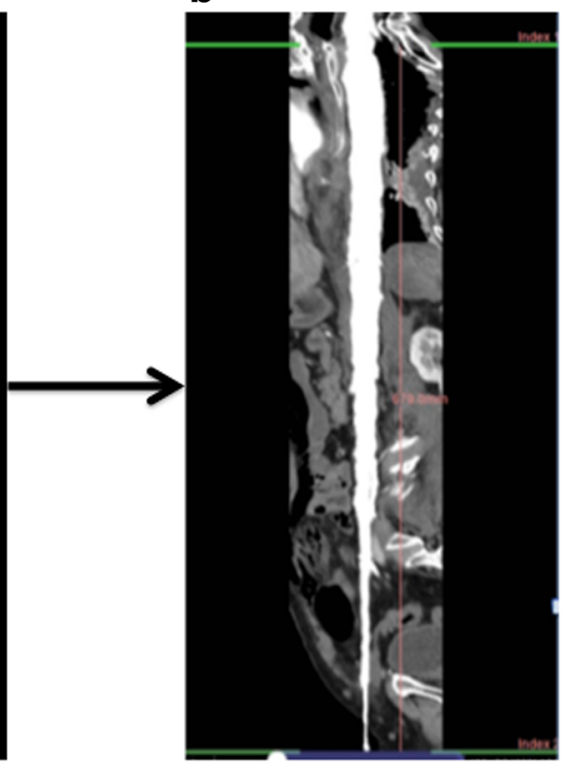

Fig. 2 How to measure the blood vessel length using Ziostation 2 PLUS. a We plotted the center point of the contrasted vascular lumen from the common femoral artery to the blood vessel cross section at the tip of REBOA at the horizontal disconnection of CT. $\mathbf{b}$ We reconstructed meandering blood vessels approximately linearly and measured the blood vessel length 


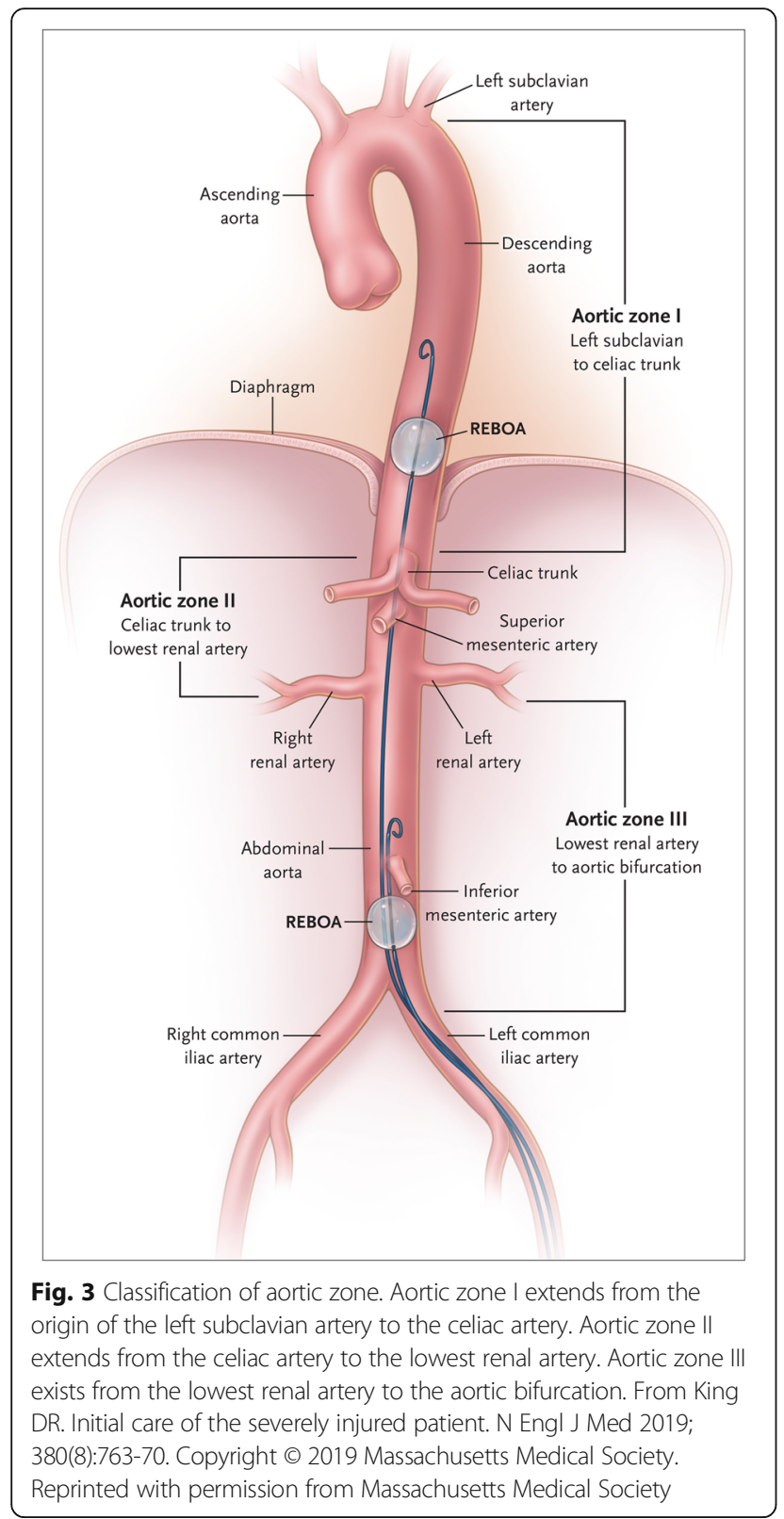

Balloon $^{\text {tw }}$ or Rescue Balloon ER ${ }^{\text {mix }}$ (Tokai Medical Products Corp., Kasugai, Aichi, Japan).

\section{Data collection}

Patient characteristics (age, sex, height, and racial group), mechanism of injury, patient vital signs, FAST whether positive or negative, injury severity score (ISS) [27], left or right approach to the common femoral artery, the aortic zone in which the REBOA tip was located, whether REBOA was inflated or deflated, and the outcome were collected from the medical records. The ISS was calculated for each patient.

\section{Statistical analysis}

We obtained descriptive statistics, such as median and interquartile range for continuous variables, and frequency and proportion for categorical variables, and compared them between the inflate and deflate groups. We used the Mann-Whitney $U$ test for continuous variables, and the Fisher exact test for categorical variables. The clinical characteristics of all included cases were described. The trace value (vascular length) by the blood vessel center line from the common femoral artery to the tip of REBOA and the length of REBOA itself (REBOA insertion length) from the common femoral artery to the tip of REBOA were divided into the inflate group and the deflate group and examined using the related-samples Wilcoxon signed-rank test. A compatibility test was conducted for statistical analysis, and twosided $p<0.05$ was regarded as significant. All analyses were performed using IBM SPSS ver. 23 (IBM Corp., Armonk, NY, USA).

\section{Results}

The median (interquartile range) age of patients was 43 years (34-59.5), and 23 patients $(85.2 \%)$ were male. There were 26 blunt traumas (96.3\%). The mechanism of the injury consisted of 10 falls $(37.0 \%), 7$ traffic injury (25.9\%), 5 pedestrian injury (18.5\%), 3 train injury (11.1\%), 1 compression (3.7\%), and 1 gunshot wound (3.7\%). The mean ISS was 31.4. Among all patients, 10 patients (37.0\%) died in the hospital (Table 1).

Table 2 shows the characteristics of 27 patients. The mean values for systolic blood pressure, heart rate, and respiratory rate were $65.8 \mathrm{mmHg}, 101.2 \mathrm{bpm}$, and 23.7/

Table 1 Characteristics of patients who were inserted with REBOA and have taken contrast CT

\begin{tabular}{lll}
\hline & Inflate $(n=11)$ & Deflate $(n=16)$ \\
\hline Age (years) & $43(29.5-59.5)$ & $43(37-57.25)$ \\
Male, $n(\%)$ & $10(90.9 \%)$ & $13(81.3 \%)$ \\
Blunt injury, $n(\%)$ & $11(100 \%)$ & $15(93.8 \%)$ \\
Mechanism of injury, $n(\%)$ & & \\
$\quad$ Fall & $3(27.3 \%)$ & $7(43.8 \%)$ \\
$\quad$ Traffic & $1(9.1 \%)$ & $6(37.5 \%)$ \\
$\quad$ Pedestrian & $4(36.4 \%)$ & $1(6.25 \%)$ \\
$\quad$ Train & $3(27.3 \%)$ & $0(0 \%)$ \\
$\quad$ Compression & $0(0 \%)$ & $1(6.25 \%)$ \\
$\quad$ Gunshot & $0(0 \%)$ & $1(6.25 \%)$ \\
ISS & $26(20.5-37.5)$ & $29(26-35)$ \\
In-hospital mortality, $n(\%)$ & $5(45.5 \%)$ & $5(31.3 \%)$ \\
Blood vessel length $(\mathrm{cm})$ & $51.4(42.1-56.6)$ & $56.2(54.5-57.2)$ \\
REBOA insertion length $(\mathrm{cm})$ & $50.3(42.3-55.0)$ & $55.2(54.2-55.6)$ \\
\hline
\end{tabular}

For age, ISS, blood vessel length, and REBOA insertion length, medians and interquartile ranges (25th-75th percentile) are shown 
Table 2 Patients included in this study

\begin{tabular}{|c|c|c|c|c|c|c|c|c|c|c|c|}
\hline No.* & Age & Sex & $\begin{array}{l}\text { Height } \\
(\mathrm{cm})\end{array}$ & $\begin{array}{l}\text { Mechanism of } \\
\text { injury }\end{array}$ & ISS & $\begin{array}{l}\text { Aortic } \\
\text { zone }^{* *}\end{array}$ & $\begin{array}{l}\text { Femoral } \\
\text { approach }\end{array}$ & $\begin{array}{l}\text { Blood vessel length } \\
(\mathrm{cm})\end{array}$ & $\begin{array}{l}\text { REBOA insertion } \\
\text { length }(\mathrm{cm})\end{array}$ & $\begin{array}{l}\text { Inflate or } \\
\text { deflate }\end{array}$ & FAST \\
\hline 1 & 70 & M & 170 & Fall & 29 & I & Left & 57.0 & 55.4 & Deflate & Positive \\
\hline 2 & 56 & M & - & Compression & 26 & I & Left & 57.2 & 55.7 & Deflate & Positive \\
\hline 3 & 18 & M & 171 & Traffic & 22 & I & Left & 57.5 & 55.1 & Deflate & Negative \\
\hline 4 & 45 & M & - & Pedestrian & 50 & I & Left & 56.2 & 55.9 & Deflate & Negative \\
\hline 5 & 34 & M & 172 & Gunshot & 22 & I & Left & 55.8 & 55.1 & Deflate & Positive \\
\hline 6 & 61 & M & 182 & Traffic & 26 & I & Left & 56.3 & 55.1 & Deflate & Positive \\
\hline 7 & 32 & M & - & Pedestrian & 50 & I & Right & 55.3 & 52.9 & Inflate & Positive \\
\hline 8 & 42 & M & - & Fall & 34 & I & Left & 57.8 & 57.4 & Deflate & Positive \\
\hline 9 & 69 & M & 163 & Traffic & 17 & I & Left & 56.7 & 56.1 & Inflate & Negative \\
\hline 10 & 38 & M & - & Fall & 29 & I & Left & 56.3 & 55.0 & Deflate & Negative \\
\hline 11 & 43 & M & 166 & Fall & 26 & I & Right & 56.2 & 55.5 & Deflate & Positive \\
\hline 12 & 43 & M & - & Traffic & 41 & I & Right & 49.9 & 49.6 & Deflate & Positive \\
\hline 13 & 39 & $F$ & - & Fall & 41 & I & Right & 55.7 & 55.3 & Deflate & Positive \\
\hline 14 & 34 & M & - & Traffic & 34 & I & Right & 54.2 & 54.0 & Deflate & Positive \\
\hline 15 & 58 & $F$ & - & Fall & 66 & I & Left & 52.4 & 50.3 & Inflate & Negative \\
\hline 16 & 61 & M & 172 & Pedestrian & 26 & I & Right & 56.6 & 55.0 & Inflate & Negative \\
\hline 17 & 43 & M & - & Train & 26 & I & Left & 42.1 & 42.3 & Inflate & Negative \\
\hline 18 & 69 & M & 173 & Traffic & 35 & I & Right & 56.8 & 55.4 & Deflate & Negative \\
\hline 19 & 27 & M & 173 & Fall & 24 & I & Left & 64.3 & 63.9 & Inflate & Negative \\
\hline 20 & 61 & M & 168 & Pedestrian & 33 & I & Right & 39.0 & 39.5 & Inflate & Positive \\
\hline 21 & 49 & M & 175 & Train & 10 & I & Left & 43.4 & 43.8 & Inflate & Negative \\
\hline 22 & 50 & M & 171 & Fall & 35 & 0 & Right & 58.8 & 58.7 & Deflate & Negative \\
\hline 23 & 19 & M & - & Traffic & 41 & I & Left & 47.5 & 47.8 & Inflate & Positive \\
\hline 24 & 88 & F & 148 & Fall & 27 & I & Right & 45.2 & 43.5 & Deflate & Negative \\
\hline 25 & 20 & M & - & Fall & 16 & I & Right & 40.5 & 41.4 & Inflate & Negative \\
\hline 26 & 42 & M & - & Train & 34 & I & Right & 51.4 & 52.0 & Inflate & Negative \\
\hline 27 & 18 & $F$ & - & Traffic & 27 & I & Left & 42.4 & 42.1 & Deflate & Positive \\
\hline
\end{tabular}

FAST focused assessment with sonography for trauma

*Case 6 was Caucasian, and all of the others were Asian

**This study defined aortic zone 0 as placement on the head side of the left subclavian artery bifurcation, which is a non-standard location. Case 22 was not inflated, and no complications occurred

min, respectively. There were 26 patients on REBOA that was placed in aortic zone I, and one had been placed on the head side of the left subclavian artery bifurcation. The insertion site of REBOA was from the right common femoral artery in 12 patients and from the left common femoral artery in 15 patients. The REBOA deflate group included 16 patients; the inflate group included 11 patients.

A total of 16 patients had deflated REBOA during CT imaging, and 13 patients (81.3\%) had low blood pressure. Six patients (37.5\%) had a head abbreviated injury score (AIS) greater than or equal to 3 . The mortality rate was $31.3 \%$ (five patients), including two patients (12.5\%) who were in cardiopulmonary arrest on arrival. The mean ISS was 31.5. There were 15 patients who had REBOA placed in aortic zone I, and the remaining 1 patient was placed on the head side from the branch of the left subclavian artery. The median trace value (interquartile range) of the blood vessel center line from the common femoral artery to the tip of REBOA (blood vessel length) and the length of REBOA itself from the common femoral artery to the tip of REBOA (REBOA insertion length) were $56.2 \mathrm{~cm}(54.5-57.2 \mathrm{~cm})$ and $55.2 \mathrm{~cm}(54.2-$ $55.6 \mathrm{~cm})$, respectively $(p<0.0001)$.

A total of 11 patients had inflated REBOA during CT imaging. Ten patients (90.9\%) had low blood pressure. In three patients $(27.3 \%)$, the head AIS was larger than or equal to 3 . The mortality rate was $45.5 \%$ (five patients), including two patients (18.2\%) who were in cardiopulmonary arrest on arrival. The mean ISS was 31.2. 
REBOA were all placed in aortic zone I. The median trace value (interquartile range) of the blood vessel center line from the common femoral artery to the tip of REBOA (blood vessel length) and the length of REBOA itself from the common femoral artery to the tip of REBOA (REBOA insertion length) were $51.4 \mathrm{~cm}(42.1-56.6 \mathrm{~cm})$ and 50.3 $\mathrm{cm}(42.3-55.0 \mathrm{~cm})(p=0.594)$, respectively.

\section{Discussion}

In our study, we reconstructed the CT data and confirmed the trajectory of REBOA in the blood vessel. We first found that compared to the center line of the blood vessel, REBOA traveled more linearly, and if the balloon was deflated when REBOA was inserted, it was placed $1.0 \mathrm{~cm}$ longer than the insertion length of REBOA. On the other hand, that was not the case when inflating the balloon. This seems to suggest that REBOA travels more linearly in meandering blood vessels. Our findings can enable practitioners to obtain a more precise REBOA placement distance, consequently leading to a safer approach that is unaffected by institutional or personnel variability.

From the results of this study, we found that it is important to always assume the possibility of placement distal to the blood vessel, rather than the assumed insertion length when inserting REBOA under nonfluoroscopy. In this study, no fatal complications accompanying placing REBOA were observed. In addition, one case in which REBOA had been detained beyond aortic zone I was also included.

A previous study established a method of using landmarks of the body surface under non-fluoroscopy when inserting REBOA. If the insertion length was longer than the length from the thigh to the xiphoid process and it is shorter than the length from the thigh to the sternum notch, REBOA was placed in aortic zone I [15]. Another study using cadavers established a method using landmarks on the body surface, in which REBOA is placed in aortic zone I when inserted from the length of the thigh to the mid-sternum [14]. The method of using landmarks on the body surface seems to be a simple and easy-to-use method that does not require special devices and knowledge. There is a possibility that it can be applied in emergency outpatient or prehospital situations in which fluoroscopy or simple X-ray are not available [28]. However, it is difficult to estimate where the actual tip of REBOA is located, because the influence of the hemodynamics and degree of balloon dilation is not taken into consideration [16]. Therefore, to understand the dynamics of REBOA in a blood vessel, this study confirmed the position of its tip in cases in which REBOA had been inserted. In the deflated group of REBOA, the median (interquartile range) of the trace value (blood vessel length) by the blood vessel center line from the common femoral artery to the tip of
REBOA and the length of REBOA itself from the common femoral artery to the tip of REBOA of the insertion length were $56.2 \mathrm{~cm}(54.5-57.2 \mathrm{~cm})$ and $55.2 \mathrm{~cm}(54.2-$ $55.6 \mathrm{~cm})(p<0.0001)$, respectively, and as a median, the difference was $1.0 \mathrm{~cm}$. This means that the tip is significantly deeper by $1.0 \mathrm{~cm}$ as a median than the assumed length, when REBOA is inserted from the common femoral artery. The possible explanation would be that the running of REBOA and the center line of the aorta would not coincide with each other. REBOA would travel in the blood vessel more linearly by entering the metallic stylet inside the catheter. In addition, there was a tendency to travel as if it touched the aorta wall in a shortcut, and as a result, it was placed at a position deeper than the assumed REBOA insertion length. In the inflated group of REBOA, the median (interquartile range) of the trace value (blood vessel length) by the blood vessel center line from the common femoral artery to the tip of REBOA and the length of REBOA itself from the common femoral artery to the tip of REBOA of the insertion length were $51.4 \mathrm{~cm}(42.1-56.6 \mathrm{~cm})$ and $50.3 \mathrm{~cm}(42.3-55.0 \mathrm{~cm})$, respectively, and there was no significant association $(p=0.594)$. The possible explanation would be that the individual difference was large to the extent that the balloon inflated and the extent to which the balloon was pushed back toward the caudal depending on the degree of blood pressure.

There are several limitations in this study to mention. First, the length of the aorta and the degree of meandering might vary depending on age, sex, race, and other factors. Second, we did not investigate how far the balloon was inflated in the REBOA inflated group. Third, the current treatment procedure may not be common at other facilities and overseas, which will affect the external validity of this study's findings. However, in several Japanese tertiary care emergency centers, REBOA was used by emergency physicians without fluoroscopic guidance and placed in a safe location with subsequent confirmation via CT [23]. Fourth, in this procedure, a wire is left at the time of measurement. There is a possibility that REBOA might be pushed distally by the flow, which could not be controlled in the current study design. Thus, further research is necessary to evaluate the flow mechanism. Fifth, since all of the cases but one were Asian, there were limitations to the external validity. Thus, the study findings might not apply to other racial groups. Finally, future international studies with larger sample sizes and more facilities are desirable to improve the representability and the generalizability of the findings.

\section{Conclusions}

If REBOA was deflated, it was placed $1.0 \mathrm{~cm}$ longer than the insertion length of REBOA itself, but that 
was not the case when inflating REBOA. The individual difference was large to the extent that the balloon inflated and the extent to which the balloon was pushed back toward the caudal depending on the degree of blood pressure. Further studies are needed to validate our findings.

\section{Abbreviations}

3D: Three-dimensional; AIS: Abbreviated injury score; CT: Computed tomography; FAST: Focused assessment with sonography for trauma; ISS: Injury severity score; IVR: Interventional radiology; REBOA: Resuscitative endovascular balloon occlusion of the aorta; YCU: Yokohama City University; YKH: Yokosuka Kyosai Hospital

\section{Acknowledgements}

Not applicable.

\section{Authors' contributions}

$\mathrm{KN}, \mathrm{HT}, \mathrm{TA}, \mathrm{TD}$, and NM designed the study. KN and TA analyzed and interpreted the data. $K N$, TA, and NM drafted the manuscript. $K N, H T, T A, K Y$, TD, IT, and NM contributed to the critical revisions. All authors read and approved the final manuscript.

\section{Funding}

Not applicable.

\section{Availability of data and materials}

The datasets used during the current study are available from the corresponding author on reasonable request.

\section{Ethics approval and consent to participate}

This study was approved by the institutional review boards at both institutes.

\section{Consent for publication}

This study was an observational study. Thus, obtaining a consent from a patient was waived by the institutional review board.

\section{Competing interests}

The authors declare that they have no competing interests.

\section{Author details}

1Advanced Critical Care and Emergency Center, Yokohama City University Medical Center, 4-57 Urafunecho, Minami-ku, Yokohama, Kanagawa 232-0024, Japan. 2Department of Emergency Medicine, Graduate School of Medicine, Yokohama City University, 3-9 Fukuura, Kanazawa-ku, Yokohama, Kanagawa 236-0004, Japan. ${ }^{3}$ Department of Surgery Intensive Care, Nippon Medical School Hospital, 1-1-5 Sendagi, Bunkyo-ku, Tokyo 113-8603, Japan. ${ }^{4}$ Critical Care and Emergency Center, Yokosuka Kyosai Hospital, Yonegahama Street 1-16, Yokosuka, Kanagawa 238-8558, Japan. ${ }^{5}$ Department of Acute Medicine, Graduate School of Medicine, The University of Tokyo, 7-3-1 Hongo, Bunkyo-ku, Tokyo 113-8655, Japan.

\section{Received: 9 May 2019 Accepted: 8 July 2019}

\section{Published online: 16 July 2019}

\section{References}

1. Pezy P, Flaris AN, Prat NJ, et al. Fixed-distance model for balloon placement during fluoroscopy-free resuscitative endovascular balloon occlusion of the aorta in a civilian population. JAMA Surg. 2017;152:351-8

2. Gamberini E, Coccolini F, Tamagnini B, et al. Resuscitative endovascular balloon occlusion of the aorta in trauma: a systematic review of the literature. World J Emerg Surg. 2017;12:42.

3. Davis JS, Satahoo SS, Butler FK, et al. An analysis of prehospital deaths: who can we save? J Trauma Acute Care Surg. 2014;77:213-8.

4. Morrison JJ, Ross JD, Houston R, Watson JD, Sokol KK, Rasmussen TE. Use of resuscitative endovascular balloon occlusion of the aorta in a highly lethal model of noncompressible torso hemorrhage. Shock (Augusta, Ga). 2014;41: 130-7.

5. Tsurukiri J, Akamine I, Sato T, et al. Resuscitative endovascular balloon occlusion of the aorta for uncontrolled haemorrhagic shock as an adjunct to haemostatic procedures in the acute care setting. Scandinavian journal of trauma, resuscitation and emergency medicine. 2016:24:13.

6. Tien HC, Spencer F, Tremblay LN, Rizoli SB, Brenneman FD. Preventable deaths from hemorrhage at a level I Canadian trauma center. The Journal of trauma. 2007;62:142-6.

7. Bansal V, Fortlage D, Lee JG, Costantini T, Potenza B, Coimbra R. Hemorrhage is more prevalent than brain injury in early trauma deaths: the golden six hours. European journal of trauma and emergency surgery: official publication of the European Trauma Society. 2009;35:26-30.

8. Inoue J, Shiraishi A, Yoshiyuki A, Haruta K, Matsui H, Otomo Y. Resuscitative endovascular balloon occlusion of the aorta might be dangerous in patients with severe torso trauma: a propensity score analysis. J Trauma Acute Care Surg. 2016;80:559-66 discussion 66-7.

9. Moore LJ, Martin CD, Harvin JA, Wade CE, Holcomb JB. Resuscitative endovascular balloon occlusion of the aorta for control of noncompressible truncal hemorrhage in the abdomen and pelvis. American journal of surgery. 2016:212:1222-30.

10. Norii T, Crandall C, Terasaka Y. Survival of severe blunt trauma patients treated with resuscitative endovascular balloon occlusion of the aorta compared with propensity score-adjusted untreated patients. J Trauma Acute Care Surg. 2015:78:721-8.

11. Brenner $M$, Teeter $W$, Hoehn $M$, et al. Use of resuscitative endovascular balloon occlusion of the aorta for proximal aortic control in patients with severe hemorrhage and arrest. JAMA Surg. 2018;153:130-5.

12. King DR. Initial care of the severely injured patient. The New England journal of medicine. 2019;380:763-70

13. Linnebur $M$, Inaba $K$, Haltmeier $T$, et al. Emergent non-image-guided resuscitative endovascular balloon occlusion of the aorta (REBOA) catheter placement: a cadaver-based study. J Trauma Acute Care Surg. 2016:81:453-7.

14. Okada Y, Narumiya H, Ishi W, liduka R. Anatomical landmarks for safely implementing resuscitative balloon occlusion of the aorta (REBOA) in zone 1 without fluoroscopy. Scandinavian journal of trauma, resuscitation and emergency medicine. 2017;25:63.

15. MacTaggart JN, Poulson WE, Akhter M, et al. Morphometric roadmaps to improve accurate device delivery for fluoroscopy-free resuscitative endovascular balloon occlusion of the aorta. J Trauma Acute Care Surg. 2016:80:941-6.

16. Wessels LE, Wallace JD, Bowie J, Butler WJ, Spalding C, Krzyzaniak M. Radiofrequency identification of the ER-REBOA: confirmation of placement without fluoroscopy. Military medicine. 2018.

17. Yokohama city homepage. http://www.city.yokohama.lg.jp/ex/stat/jinko/ news-e.html. Accessed 1 Jan 2019.

18. Yokosuka city homepage. https://www.city.yokosuka.kanagawa.jp/0830/ data/toukei/suikei/now.html. Accessed 1 Jan 2019.

19. Zushi city homepage. https://www.city.zushi.kanagawa.jp/syokan/kikaku/ population-data.html. Accessed 1 Jan 2019.

20. Miura city homepage. http://www.city.miura.kanagawa.jp/hisho/eng/ information.html. Accessed 1 Jan 2019.

21. van der Burg BLS B, van Dongen T, Morrison JJ, et al. A systematic review and meta-analysis of the use of resuscitative endovascular balloon occlusion of the aorta in the management of major exsanguination. European journal of trauma and emergency surgery: official publication of the European Trauma Society. 2018;44:535-50.

22. Stannard A, Eliason JL, Rasmussen TE, Resuscitative endovascular balloon occlusion of the aorta (REBOA) as an adjunct for hemorrhagic shock. The Journal of trauma. 2011:71:1869-72.

23. Sato R, Kuriyama A, Takaesu R, et al. Resuscitative endovascular balloon occlusion of the aorta performed by emergency physicians for traumatic hemorrhagic shock: a case series from Japanese emergency rooms. Critical care (London, England). 2018;22:103.

24. Cheema F, Garcia C, Rivera AG, Chao E. CE: The use of resuscitative endovascular balloon occlusion of the aorta in treating hemorrhagic shock from severe trauma. The American journal of nursing. 2018:118:22-8.

25. Hoehn MR, Hansraj NZ, Pasley AM, et al. Resuscitative endovascular balloon occlusion of the aorta for non-traumatic intra-abdominal hemorrhage. European journal of trauma and emergency surgery: official publication of the European Trauma Society. 2018.

26. Zakaluzny SA, Beldowicz BC, Salcedo ES, JJ DB, Moore LJ, Brenner M. Guidelines for a system-wide multi-disciplinary approach to institutional REBOA implementation. J Trauma Acute Care Surg. 2018. 
27. Baker SP, O'Neill B, Haddon W Jr, Long WB. The injury severity score: a method for describing patients with multiple injuries and evaluating emergency care. The Journal of trauma. 1974;14:187-96.

28. Richardson R, Adcock L. CADTH Rapid Response Reports. Resuscitative endovascular balloon occlusion of the aorta for control of non-compressible truncal hemorrhage: a review of clinical effectiveness and guidelines.

Ottawa: Canadian Agency for Drugs and Technologies in Health. Copyright

(c) 2018 Canadian Agency for Drugs and Technologies in Health; 2018.

\section{Publisher's Note}

Springer Nature remains neutral with regard to jurisdictional claims in published maps and institutional affiliations.

Ready to submit your research? Choose BMC and benefit from:

- fast, convenient online submission

- thorough peer review by experienced researchers in your field

- rapid publication on acceptance

- support for research data, including large and complex data types

- gold Open Access which fosters wider collaboration and increased citations

- maximum visibility for your research: over $100 \mathrm{M}$ website views per year

At $B M C$, research is always in progress.

Learn more biomedcentral.com/submissions 